Results The overview reveals that the external eye physiology (separate from internal eye effects), eye diseases (evaporative dry eye, aqueous-deficient dry eye, and gland dysfunctions) aggravate the stability of precorneal tear film (PTF); this appears to cause hyperosmolarity and initiation of inflammatory reactions. Further, indoor environmental, occupational and personal risk factors may aggravate the PTF stability. These are, inter alia age, contact lenses, cosmetics, diet, draft, gender, low humidity and high temperature, medication, outdoor and combustion pollutants, and VDU work. Psychological stressors may further influence the reporting behaviour of symptoms. The risk factor impact may occur in a combined and exacerbating manner.

Discussion Multifactorial causalities show two key indoor parameters, visually demanding tasks combined with low relative humidity. A substantial fraction of office workers may have unrecognised DE diseases which may further be exacerbated by environmental, occupational and personal risk factors. Dry eye-like related symptoms in office-like environments may be considered a subgroup of 'dry eye' disease.

A number of other risk factors in the office environment are associated with reported DE symptoms. These may in combination exacerbate the symptom reporting. Except for combined events, indoor VOCs, formaldehyde, and acrolein are not expected to cause sensory-related eye symptoms in offices without aggravation of the PTF.

\section{$1688 \mathrm{C}$ THE CONTRIBUTION OF TASK- AND WORKER-RELATED ACCOMMODATION TO ASTHENOPIA: A CRITICAL LITERATURE REVIEW}

${ }^{1}$ Bruno Piccoli*2Dino Pisaniello, ', Letizia Colais ${ }^{2}$ Sharyn Gaskin, ${ }^{3}$ Marco D'Orso. ${ }^{1}$ Institute of Public Health - Section of Occupational Health, Catholic University of the Sacred Heart, Rome, Italy; ${ }^{2}$ School of Public Health, University of Adelaide, Adelaide, Australia; ${ }^{3}$ School of Medicine, University of Milan - Bicocca, Monza, Italy

\subsection{6/oemed-2018-ICOHabstracts. 1528}

Introduction Near work is believed to be a key factor in the development of occupational asthenopia as well as being a possible cofactor for the development of adult myopia. Prolonged viewing distances of less than one metre induce visual symptoms by imposing a sustained contraction of ciliary muscles. Small screens on mobile devices are very common in modern work environments, and there are many jobs and tasks that require near work every day. Furthermore, there is an ageing workforce where there is less capacity for visual accommodation. This review critically assesses the published literature on asthenopia attributable to task observation distance and worker-related accommodative capacity.

Methods A systematic search of PubMed, Scopus, and Embase was used, and supplemented with forwards, backwards and handsearching, including by author. The major search terms were 'observation distance', 'near work' and 'visual work load'.

Results Most published papers reporting on asthenopia in the workplace classify visual effort in broad terms, without objective 'on site' measurement of task-determined visual distances/ durations. Similarly, few papers separately consider workers over 45 (presbyopia), and those who are long sighted (hyperopia), where there is a greater requirement of accommodation compared to normal sighted (emmetropes). Even fewer papers juxtapose the observation distance with the worker capacity for accommodation, i.e. combining exposure and susceptibility, which is essential for the characterisation of asthenopia risk.
Discussion In order to assess and manage risks for asthenopia attributable to visual effort, equal weight should be given to task and individual factors. Future studies should utilise objective measurements of task viewing distance and durations. Currently, there is a need for a personal monitoring device that can continuously measure and datalog these parameters. With regard to an ageing workforce, intervention research is needed to determine whether the provision of larger screens, larger images and less visual demanding software reduces asthenopia.

\section{IS HEAVY VDT USE A RISK FOR VISUAL FIELD ABNORMALITIES AMONG JAPANESE WORKERS?}

${ }^{1} \mathrm{M}$ Tatemichi ${ }^{*},{ }^{2} \mathrm{~T}$ Nakagawa, ${ }^{2} \mathrm{~T}$ Honda, ${ }^{1} \mathrm{~S}$ Owada, ${ }^{1} \mathrm{H}$ Endo, ${ }^{2} \mathrm{~T}$ Hayashi, ${ }^{3} \mathrm{~T}$ Nakano. ${ }^{1}$ Dep. of Preventive Medicine, Tokai University School of Medicine, Kanagawa, Japan; ${ }^{2}$ Hitachi Health Centre, Ibaraki, Japan; ${ }^{3}$ Dep. of Ophthalmology, The Jikei University, Tokyo, Japan

10.1136/oemed-2018-ICOHabstracts. 1529

Introduction The rapid global spread of information technology (IT), and most recently of new media (e.g., smartphones and tablets), has continually increased exposure to visual display terminals (VDT). This exposure is particularly marked among general workers. The health issues related to long-time VDT use are of great concern. Our previous large-scale, population-based study using frequency doubling technology perimetry (FDT) perimetry in the workplace indicated a possible association between a history of VDT use and glaucoma. Interestingly, we observed a significant interaction effect of myopia on the association between computer use and glaucoma; specifically, an increased risk of glaucomatous visual field abnormality (VFA) was observed only among frequent VDT users with myopia (J Epidemiol Community Health, 2004;58 (12):1021-7. etc.). However, those studies featured a crosssectional design. We designed a retrospective cohort study to investigate the association between computer use and VFA using the FDT test.

Methods The study included 2377 workers (mean age 45.7 \pm 8.3 years) who initially exhibited no VFA during FDT testing. Subjects then underwent annual follow-up FDT testing for 7 years, and VFA were determined by using a FDT-test protocol (FDT-VFA). Subjects with FDT-VFA were examined by ophthalmologists. Baseline data about the mean duration of computer years during a 5 year period and refractive errors were obtained via self-administered questionnaire.

Results A Cox proportional hazard analysis demonstrated that heavy VDT users ( $>8 \mathrm{~h} /$ day) had a significantly increased risk of FDT-VFA (hazard ratio $(\mathrm{HR})=2.85,95 \%$ confidence interval (CI): $1.26-6.48)$ relative to light users $(<4 \mathrm{~h} /$ day), and this association was strengthened among subjects with refractive errors $(\mathrm{HR}=4.48,95 \% \mathrm{CI}: 1.87$ to 10.74). The VDT usage history also significantly correlated with FDT-VFA among subject with refractive errors $(p<0.05)$, and $73.1 \%$ of subjects with FDT-VFA and refractive errors were diagnosed as glaucomatous.

Conclusion The incidence of FDT-VFA appears to increase among Japanese workers who are heavy VDT users, particularly if they have refractive errors. These results are consistent with findings of our previous studies. Further investigations of epidemiology and causality are warranted, because causal relationship between VDT use and glaucoma is unknown. 